\title{
Pacific
}

Journal of

Mathematics

\section{AUTOMATIC CONTINUITY FOR WEAKLY DECOMPOSABLE OPERATORS}

Ridgley LANGe, Sheng-WANG WANG AND Yong ZhONG 


\title{
AUTOMATIC CONTINUITY FOR WEAKLY DECOMPOSABLE OPERATORS
}

\author{
R. LANGE, S. WANG AND Y. ZHONG
}

\begin{abstract}
The authors introduce a new class of operators that are weakly decomposable relative to the identity, and some of their properties are derived; for example, these operators have the single valued extension property. The main result is that every generalized intertwining of an operator having property $(\delta)$ with such a weakly decomposable one is necessarily bounded whenever certain side conditions are satisfied. Examples also show that this class of weakly decomposable operators is not comparable by inclusion to the classical cases (e.g. decomposable operators).
\end{abstract}

\section{Introduction.}

In this note we shall generalize some results of Laursen and Neumann [13] on the automatic continuity of intertwinings of operators with certain spectral decomposition properties. Specifically, we prove that a linear map which is a generalized intertwining of an operator satisfying property $(\delta)$ with a second admissible operator that is weakly decomposable relative to the identity (WDI) is necessarily continuous (bounded) (provided the operator pair has no critical eigenvalue). The properties $(\delta)$ and WDI are both relaxations of the notion "decomposable" but in different directions; see below for details. We mention that some of our results and proofs have been improved and shortened by appeals to [13], which appeared after the original submission of the present paper.

Section 2 of the paper deals with definitions and other background needed for this study. Some results are proved which have their own independent interest. For example, we show that the notions of "admissible" and "subadmissible"operator (see $[\mathbf{1 3}, \mathbf{1 5}]$ ) are identical. Our Proposition $2.1 \mathrm{seems}$ to be known, but the authors do not know where it may have been published.

In Section 3 we give the definition of our new "weakly" decomposable operators and then establish some of their elementary properties, among which is the single-valued extension property. We show through two examples that, unlike other subclasses with spectral decomposition, our new class is not comparable by inclusion to the class of decomposable operators. 
In Section 4 we prove our main theorem (Theorem 4.1). The crucial result here is the admissibility of the WDI operator mentioned above. The last section gives some applications. We also want to thank the referee for helpful suggestions in revising this paper.

\section{Preliminaries.}

In this section we state some definitions and notations used in the paper. We also prove that the notions "subadmissible" and "admissible", introduced in $[13,15]$, resp., are the same.

We write $T \in L(X)$ to mean that $\mathrm{T}$ is a bounded linear operator on the complex Banach space X, while $\sigma(T)$ and $\rho(T)$ denote its spectrum and resolvent set, resp. We recall that the analytic spectral manifolds for $\mathrm{T}$ are defined for the set $\mathbf{F}$ in the plane $\mathbf{C}$ by the formula

$$
X(T, F)=\left\{x \in X: \sigma_{T}(x) \subset F\right\}
$$

where $\sigma_{T}(x)$ denotes the local spectrum of $\mathrm{T}$ at $\mathrm{x}$ which itself is defined as the complement of the union of all the open sets in $\mathbf{C}$ on which are defined local analytic solutions of the equation $(\lambda-T) f(\lambda)=x$. We say that $\mathrm{T}$ has the single valued extension property (SVEP) if an analytic function $f: D \rightarrow X$ vanishes on $\mathrm{D}$ whenever it satisfies $(\lambda-T) f(\lambda)=0$ on $\mathrm{D}$.

Proposition 2.1. If $X(T, F)$ is closed for every closed set $F$, then $T$ has SVEP.

To prove Proposition 2.1 we need several lemmas.

Lemma $2.2\left[10\right.$, p. 16]. If $F$ is closed and $\lambda_{0} \in F, x_{0} \in X$, and if $\left(\lambda_{0}-\right.$ $T) x_{0} \in X(T, F)$, then $x_{0} \in X(T, F)$.

Proof. Let $\mathrm{f}$ be an analytic $\mathrm{X}$-valued function on some open set $\mathrm{D}$ with $D \cap F=\varnothing$ such that $(\lambda-T) f(\lambda)=\left(\lambda_{0}-T\right) x_{0}$ for $\lambda \in D$. Then the function $h(\lambda)=\left(\lambda-\lambda_{0}\right)^{-1}\left[x_{0}-f(\lambda)\right]$ satisfies

$$
(\lambda-T) h(\lambda)=\left(\lambda-\lambda_{0}\right)^{-1}\left[\left(\lambda-\lambda_{0}\right) x_{0}+\left(\lambda_{0}-T\right) x_{0}-(\lambda-T) f(\lambda)\right]=x_{0}
$$

for $\lambda \in D$, which shows that $x_{0} \in X(T, F)$, since D is an arbitrary open set disjoint from $\mathrm{F}$.

Lemma 2.3. Let $F$ be a fixed closed set. If $X(T, F)$ is closed, then

$$
\partial \sigma(T \mid X(T, F)) \subset F
$$


where $\partial S$ denotes the boundary of $S$.

Proof. Assume there exists $\lambda_{0} \in \partial \sigma(T \mid X(T, F))$ but $\lambda_{0} \not \notin F$. Then $\left(\lambda_{0}-\right.$ $T) \mid X(T, F)$ is surjective, and since spectra are closed we infer that $\left(\lambda_{0}-\right.$ $T) \mid X(T, F)$ is not injective. By [6, Corollary 2.4], $T \mid X(T, F)$ does not have SVEP. Thus [6, Cor. 2.3] ensures existence of a neighborhood $\delta$ of $\lambda_{0}$ and a nonzero analytic function $\mathrm{f}$ defined on $\delta$ with values in $\mathrm{X}$ such that

$$
(\lambda-T) f(\lambda)=0 \quad(\lambda \in \delta) .
$$

We have $\rho(T \mid X(T, F)) \cap \delta \neq \varnothing$ because $\lambda_{0} \in \partial \sigma(T \mid X(T, F))$. On other hand, (2.1) implies that $T \mid X(T, F)$ has eigenvalues in $\rho(T \mid X(T, F)) \cap \delta$, a contradiction which proves the lemma.

Corollary 2.4. If $F$ and $X(T, F)$ are closed, then $\sigma(T \mid X(T, F)) \subset \widehat{F}$, where $\widehat{F}$ is the union of $F$ and all bounded components of its complement.

Lemma 2.5. Suppose $D \subset \mathbf{C}$ is open and connected. If the equation $(\lambda-$ $T) f(\lambda)=0$ has a nonzero analytic solution on $D$ and if $X(\mathrm{~T}, F)$ is closed for each closed $F$ with $F \cap D \neq \varnothing$, then $D \subset \sigma(T \mid X(T, F))$.

Proof. Let $\lambda_{0} \in F \cap D$. By Lemma 2.2, $f\left(\lambda_{0}\right) \in X(T, F)$. Moreover, since $T f(\lambda)=\lambda f(\lambda)$ for all $\lambda \in D$, differentiation yields $T f^{(n)}(\lambda)=\lambda f^{(n)}(\lambda)+$ $n f^{(n+1)}(\lambda)$ for all $\lambda \in D$. In particular, $\left(\lambda_{0}-T\right) f^{(n)}\left(\lambda_{0}\right)=-n f^{(n-1)}\left(\lambda_{0}\right)$. From Lemma 2.2 it follows again by an easy inductive argument that $f^{(n)}\left(\lambda_{0}\right)$ $\in X(T, F)$ for $n=0,1, \ldots$. If $\left\{\lambda:\left|\lambda-\lambda_{0}\right|<\eta\right\} \subset D$, then

$$
f(\lambda)=\sum_{n=0}^{\infty} \frac{1}{n !} f^{(n)}\left(\lambda_{0}\right)\left(\lambda-\lambda_{0}\right)^{n}
$$

lies in $\mathrm{X}(\mathrm{T}, \mathrm{F})$ for all $\lambda$ with $\left|\lambda-\lambda_{0}\right|<\eta$. Hence $f(\lambda) \in X(T, F)$ for all $\lambda \in D$ by analytic continuation. Since $\mathrm{f}$ is nonzero and $(\lambda-T) f(\lambda)=0$, it follows that $D \subset \sigma(T \mid X(T, F))$.

Proof of Proposition 2.1. Suppose that $(\lambda-T) f(\lambda)=0$ for some analytic Xvalued function $\mathrm{f}$ on an open connected set $\mathrm{D}$. If $f\left(\lambda_{0}\right) \neq 0$ for some $\lambda_{0} \in D$, then choose $\eta>0$ so that $f(\lambda) \neq 0$ on the disc $\left|\lambda-\lambda_{0}\right|<\eta$ lying in D. Put $D_{0}=\left\{\lambda:\left|\lambda-\lambda_{0}\right| \leq \eta / 2\right\}$. Then $\sigma\left(T \mid X\left(T, D_{0}\right)\right) \subset D_{0}$ by Corollary 2.4. But Lemma 2.5 implies $D \subset \sigma(T \mid X(T, F))$, or $D \subset D_{0}$, a contradiction. The result follows.

Lemma 2.6. Assume that $Y$ is an invariant subspace of $T$. If $X(\mathrm{~T}, F)$ is closed for every closed $F$, then $Y(T \mid Y, F)$ is also closed.

Proof. By Proposition $2.1 \mathrm{~T}$ has SVEP, hence the conclusion follows from [19, Lemma 1.9]. 
We now recall that $\mathrm{T}$ is decomposable [6] (resp. weakly decomposable [7]) if for every open cover $\left\{G_{i}: 1 \leq i \leq n\right\}$ of the complex plane $\mathbf{C}$ there are T-invariant subspaces $M_{1}, M_{2}, \ldots, M_{n}$ such that $\sigma\left(T \mid M_{i}\right) \subset G_{i}$ for each $i$ and $X=M_{1}+\cdots+M_{n}$ (resp. $\mathrm{X}$ is the closed span of the $M_{i}$ ). If $\mathrm{T}$ is decomposable, then $\mathrm{X}(\mathrm{T}, \mathrm{F})$ is closed for closed $\mathrm{F}$. The operator $\mathrm{T}$ is called quasidecomposable [7] if it is weakly decomposable and $\mathrm{X}(\mathrm{T}, \mathrm{F})$ is closed whenever $\mathrm{F}$ is.

Following [13] and its references $[\mathrm{AE}],[\mathrm{E}]$, we say that an operator $T \in$ $L(X)$ has property $(\delta)$ if for every open cover $\{U, V\}$ of $\mathbf{C}$ and for every $x \in X$ there exist a pair of elements $u, v \in X$ and a pair of analytic functions $f: \mathbf{C} \backslash U^{-} \rightarrow X$ and $g: \mathbf{C} \backslash V^{-} \rightarrow X$ such that

$$
\begin{gathered}
x=u+v, \\
u=(\lambda-T) f(\lambda) \quad \text { for all } \lambda \in \mathbf{C} \backslash U^{-}, \\
v=(\lambda-T) g(\lambda) \quad \text { for all } \lambda \in \mathbf{C} \backslash U^{-} .
\end{gathered}
$$

The following notion of generalized intertwining can be found in [15] (see also [5, p. 48]). Let $T \in L(X)$ and $S \in L(Y)$, and let $\theta: X \rightarrow Y$ be a linear map. Let $C(S, T) \theta=S \theta-\theta T$ and define recursively

$$
C^{n}(S, T) \theta=C^{n-1}(S, T)(S \theta-\theta T)=\sum_{k=0}^{n}\left(\begin{array}{l}
n \\
k
\end{array}\right) S^{k} \theta(-T)^{n-k}
$$

for all $n$. If

$$
\left\|C^{n}(S, T) \theta\right\|^{1 / n} \rightarrow 0 \quad \text { as } n \rightarrow \infty,
$$

we shall say that $\theta$ is a generalized intertwining of $\mathrm{T}$ with $\mathrm{S}$. Clearly (2.2) contains the tacit assumption that $C^{n}(S, T) \theta$ is continuous for some, and hence almost all, $n$.

If $X=Y$ and $S=T$, then we write $C^{n}(T)$ for $C^{n}(T, T)$. According to $[4$, p. 26], $\mathrm{T}$ is called well-decomposable (WD) if for each open cover $\{U, V\}$ of $C$ there exist $P \in L(X)$ and T-invariant subspaces $\mathrm{Y}$ and $\mathrm{Z}$ such that $P X \subset Y,(I-P) X \subset Z, \sigma(T \mid Y) \subset U, \sigma(T \mid Z) \subset V$ and $C^{n}(T) P=0$ for some $n$.

The question of automatic continuity is whether a generalized intertwining of $\mathrm{T}$ with $\mathrm{S}$ is continuous under certain conditions. This question is intimately related to the notion of "algebraic spectral subspace" [11]. For $T \in L(X)$, define $E_{T}(F)$ to be the maximal linear manifold $M \subset X$ such that $(\lambda-T) M=M$ for all $\lambda \notin F ; E_{T}(F)$ is called an "algebraic spectral subspace" of T. In general, $E_{T}(F)$ need not be closed in X even if $\mathrm{F}$ is closed and $\mathrm{T}$ is decomposable [13], but we will be especially interested in the case where it is closed. 
We say that $T \in L(X)$ is admissible $[\mathbf{1 3}, \mathbf{1 5}]$ if $E_{T}(F)$ is closed whenever $\mathrm{F}$ is closed; T is said to be subadmissible [13] if there exist admissible $S \in L(Z)$ for some Banach space $\mathrm{Z}$ and a continuous linear injection $i: X \rightarrow Z$ with closed range such that $S i=i T$.

Proposition 2.7. "Admissible" and "subadmissible" are equivalent.

Proof. Obviously we need only show that every subadmissible operator is admissible. Let $\mathrm{T}$ be subadmissible with $\mathrm{S}, \mathrm{Z}$ and $\mathrm{i}$ as above. Let $Y=i(X)$. Then $\mathrm{Y}$ is a closed subspace of $\mathrm{Z}$ and S-invariant because of $S \mid Y=i T i^{-1}$. As $\mathrm{S}$ is admissible, $E_{S}(F)$ is closed and hence $E_{S}(F)=Z(S, F)$ for every closed $\mathrm{F}$ by $[15$, p. 214]. By Lemma $2.6 Y(S \mid Y, F)$ is closed. Moreover, $\mathrm{S}$ has the SVEP by Proposition 2.1 and hence $E_{S \mid Y}(F)=Y(S \mid Y, F)$ by [13, Lemma 1]. Then it is closed and so $S \mid Y$ is admissible. Hence $\mathrm{T}$ is also admissible because $T=i^{-1}(S \mid Y) i$.

Remark. Proposition 2.7, which improves [13, Proposition 2], can be proved in another way. By [11, Corollary 3.6], if $E_{S}(F)$ is closed for every closed F, then S has the SVEP. Applying [15, p. 214], [19, Lemma 1.9] and [13, Lemma 1], we can prove that $S \mid Y$ is admissible, and hence also $\mathrm{T}$.

We close this section with some other terminology used below. The operator T is algebraic if $p(T)=0$ for some nonzero polynomial $p(\lambda)$. We say that the operator pair (T,S) has critical eigenvalue $\lambda$ if $\lambda$ is an eigenvalue of $\mathrm{S}$ and the dimension of the factor space $X /(\lambda-T) X$ is infinite.

\section{Weak decomposability relative to the identity.}

Definition 3.1. Let $T \in L(X)$. Then $\mathrm{T}$ is weakly decomposable relative to the identity if for each finite open cover $\left\{G_{i}: 1 \leq i \leq n\right\}$ of the complex plane

(i) there exist T-invariant subspaces $M_{1}, M_{2}, \ldots, M_{n}$ such that $\sigma\left(T \mid M_{i}\right) \subset$ $G_{i}$ for each $i$ and

(ii) for each pair $(j, i), i=1,2, \ldots, n, j=1,2, \ldots$, there is an operator $P_{j i}$ in the commutant $\{T\}^{\prime}$ such that

(a) $I=\mathrm{WOT}-\lim _{j}\left(\sum_{i} P_{j i}\right)$,

(b) $P_{j i} X \subset M_{i}(1 \leq i \leq n$, all $j)$ where in (a) the limit is that in the weak operator topology of $\mathrm{L}(\mathrm{X})$. For brevity we call $\mathrm{T}$ a WDI operator on $\mathrm{X}$.

Theorem 3.2. Every WDI operator has SVEP.

Proof. Let T be WDI on X, and let $f: D \rightarrow X$ be analytic satisfying $(\lambda-T) f(\lambda)=0$ for all $\lambda \in D$. We may clearly suppose that $\mathrm{D}$ is connected. 
Next let $G_{1}, G_{2}$ be disjoint open discs in $\mathrm{D}$, and let $H_{1}$ be open such that $\left\{G_{1}, H_{1}\right\}$ covers $\mathbf{C}$ and $G_{1} \backslash H_{1}^{-} \neq \varnothing$. By Definition 3.1 there are T-invariant subspaces $M_{1}, N_{1}$ such that

$$
\sigma\left(T \mid M_{1}\right) \subset G_{1} \text { and } \sigma\left(T \mid N_{1}\right) \subset H_{1}
$$

and sequences $P_{j}, Q_{j} \in\{T\}^{\prime}$ such that

$$
\left\langle x-P_{j} x-Q_{j} x, u\right\rangle<j^{-1} \text { and } P_{j} X \subset M_{1} \text { and } Q_{j} X \subset N_{1}
$$

for all $x \in X, u \in X^{*}$. Since $(\lambda-T) Q_{j} f(\lambda)=0$ for $\lambda$ in $G_{1} \backslash H_{1}^{-}$, we clearly have $Q_{j} f(\lambda)=0$ for all such $\lambda$. Hence $\left\langle f(\lambda)-P_{j} f(\lambda), u\right\rangle \rightarrow 0$ as $j \rightarrow \infty$, and thus $f(\lambda)$ lies in the weak closure of $M_{1}$. Because $M_{1}$ is convex, $f(\lambda) \in M_{1}$, so $f(\lambda) \in M_{1}$ for all $\lambda \in D$ by analytic continuation. We can similarly find $M_{2}$ with $\sigma\left(T \mid M_{2}\right) \subset G_{2}$ and $f(\lambda) \in M_{2}$ for all $\lambda \in D$. Since the set $G_{1}$ and $G_{2}$ are convex, $\sigma\left(T \mid M_{1} \cap M_{2}\right) \subset G_{1} \cap G_{2}=\varnothing$, hence $M_{1} \cap M_{2}$ is the zero subspace and $f=0$ on $D$. This completes the proof.

Theorem 3.3. If $T$ is WDI on $X$, then the manifold $X(T, F)$ is closed whenever $F$ is closed.

Proof. For $\lambda$ in the complement of F define

$$
\begin{aligned}
& G_{\lambda}=\{\mu:|\mu-\lambda|<(1 / 2) \operatorname{dist}(\lambda, F)\}, \\
& H_{\lambda}=\{\mu:|\mu-\lambda|>(1 / 3) \operatorname{dist}(\lambda, F)\} .
\end{aligned}
$$

Since $\left\{G_{\lambda}, H_{\lambda}\right\}$ covers $\mathbf{C}$, let $X_{\lambda}$ and $Y_{\lambda}$ be T-invariant subspaces such that $\sigma\left(T \mid X_{\lambda}\right) \subset H_{\lambda}$ and $\sigma\left(T \mid Y_{\lambda}\right) \subset G_{\lambda}$, and let $P_{j}, Q_{j} \in\{T\}^{\prime}$ satisfy Definition 3.1(ii). We prove $X(T, F) \subset X_{\lambda}$. For if $x \in X(T, F)$, then $\left\langle x-P_{j} x-\right.$ $\left.Q_{j} x, u\right\rangle \rightarrow 0$ as $j \rightarrow \infty$ for all $u \in X^{*}$. But $Q_{j} x \in Y_{\lambda}$ for all $j$. Now $\sigma_{T}(x)$ exists because of Theorem 3.2, so for each $j$

$$
\sigma_{T}\left(Q_{j} x\right) \subset \sigma_{T}(x) \cap G_{\lambda} \subset F \cap G_{\lambda}=\varnothing .
$$

Hence $Q_{j} x=0[5$, p. 2] and thus $x$ lies in the weak (and hence norm) closure of $X_{\lambda}$ (as in Theorem 3.2). Moreover, we have $X(T, F) \subset \cap\left\{X_{\lambda}: \lambda \notin F\right\}$. On the other hand, if $x_{1}$ lies in the last intersection, then $\sigma_{T}\left(x_{1}\right) \subset H_{\lambda}$ (all $\lambda \notin F)$. So $\sigma_{T}\left(x_{1}\right) \subset \cap\left\{H_{\lambda}: \lambda \notin F\right\}=F$, i.e. $x_{1} \in X(T, F)$. Hence $X(T, F)$, as the intersection of closed subspaces, is closed itself.

Remark. The reader may wonder why the results of $\S 2$ were not applied to prove Theorem 3.3. The reason is that the defining properties of WDI operators do not allow us to conclude directly that the manifolds $\mathrm{X}(\mathrm{T}, \mathrm{F})$ are closed for closed F. 
Corollary 3.4. Every WDI operator is quasidecomposable.

Proof. Let T be WDI on X. By [7] it suffices to show that X is closed span of $X\left(T, G_{i}^{-}\right)$where $\left\{G_{i:} 1 \leq i \leq n\right\}$ is an open cover of $\mathbf{C}$. But Definition 3.1 shows that the manifold $X\left(T, G_{1}^{-}\right)+\cdots+X\left(T, G_{n}^{-}\right)$is weakly dense in $\mathrm{X}$, hence it is also norm dense.

Example 3.5. In [2] E. Albrecht constructed a certain $l^{1}$ sum of function spaces, and he proved that multiplication by the independent variable is quasidecomposable on this space but not decomposable. Here we sketch a proof that Albrecht's example is in fact a WDI operator, i.e. there exist sufficiently many operators in its commutant to guarantee part (ii) of Definition 3.1.

Let $\mathrm{D}$ be the closed unit disc, and let $B^{0}(D)$ be the set of all continuous complex-valued functions on D. Now let $B^{1}(D)$ consist of those $f \in B^{0}(D)$ which have a $\bar{\partial}$-distributional derivative $g \in B^{0}(D)$ (here $\bar{\partial}$ is the differential operator $\bar{\partial}=(1 / 2)(\partial / \partial x+i \partial / \partial y))$. Next inductively define $B^{j}(D), j=$ $2,3, \ldots$, in a similar way. With the usual Montel norm each $B^{j}(D)$ is a Banach space. Let $\mathrm{X}$ be the $l^{1}$-direct sum of all $B^{j}(D), j=0,1, \ldots$, and define $\mathrm{T}$ on $\mathrm{X}$ by formula

$$
T\left(f_{j}(\lambda)\right)=\left(\lambda f_{j}(\lambda)\right), \quad \lambda \in D .
$$

By a rather long argument Albrecht proved that $\mathrm{T}$ is quasidecomposable (see [2]).

To see that $\mathrm{T}$ is WDI, let $\left\{G_{1}, \ldots, G_{m}\right\}$ be an open cover of D (or C) and choose a system of $C^{\infty}$-functions $\left\{\phi_{k}\right\}$ with $\operatorname{supp} \phi_{k} \subset G_{k}^{-}$and $\phi_{1}+\phi_{2}+$ $\cdots+\phi_{m}=1$ on D. For $\left(f_{j}\right) \in X$ and $n=0,1, \ldots$, define

$$
f_{j}^{(n)}= \begin{cases}f_{j} & \text { if } j \leq n, \\ 0 & \text { if } j>n .\end{cases}
$$

Then clearly $\left(f_{j}^{(n)}\right) \rightarrow\left(f_{j}\right)$ in the norm of $\mathrm{X}$ as $n \rightarrow \infty$. Also for $1 \leq k \leq m$ and $n=0,1, \ldots$, define the multiplier-truncation operator $P_{k n}$ by $P_{k n}\left(f_{j}\right)=$ $\left(\phi_{k} f_{j}^{(n)}\right)$. By Albrecht's results $P_{k n} \in\{T\}^{\prime}$ and

$$
\left(\sum_{k=1}^{m} P_{k n}\right)\left(f_{j}\right)=\left(f_{j}^{(n)}\right) \rightarrow\left(f_{j}\right),
$$

that is, the sums $\sum_{k} P_{k n}$ tend to the identity in the strong operator topology, so also (WOT). Finally, the condition supp $\phi_{k} \subset G_{k}^{-}$implies that $P_{k n} X \subset$ $X\left(T, G_{k}^{-}\right)$for each $k=1,2, \ldots$. Hence $3.1(\mathrm{ii})$ is satisfied, so $\mathrm{T}$ is weakly decomposable relative to the identity. 
Example 3.6. In [1] Albrecht gave another example of an operator $T=$ $S+Q$ where $\mathrm{S}$ is generalized scalar [5] and $Q \in\{S\}^{\prime}$ and $Q^{2}=0$. In fact, he proved that each $V \in\{T\}^{\prime}$ has zero square and all their products vanish. It follows that $\mathrm{T}$ is decomposable, but $\mathrm{T}$ is not WDI. For if Definition 3.1 were satisfied, then for $j=1,2, \ldots$, there are $P_{j 1}, P_{j 2} \in\{T\}^{\prime}$ such that $V_{j}=P_{j 1}+P_{j 2} \rightarrow I$ (WOT). For $j=k$ fixed, choose $x \in X, u \in X^{*}$ with $\left\langle V_{k} x, u\right\rangle=1$. But then $0=\left\langle V_{j} V_{k} x, u\right\rangle \rightarrow\left\langle V_{k} x, u\right\rangle=1(j \rightarrow \infty)$, so $\mathrm{T}$ is not WDI.

Examples 3.5 and 3.6 show that the classes of WDI and decomposable operators are not comparable by inclusion; this fact distinguishes WDI operators from all other previously studied classes of operators with spectral decomposition, which include well-decomposable operators.

It is easy to see that a WDI operator having property $(\delta)$ is decomposable. On the other hand, if $\mathrm{T}$ is the operator given by Albrecht in [3], then by the argument of $[3$, p. $12(\mathrm{~B})] \mathrm{T}$ has no nontrivial spectral maximal space contained in $X(T,[0,1])$, i.e. every spectral maximal space contained in $X(T,[0,1])$ is either $\{0\}$ or $X(T,[0,1])$ itself. Now if $\mathrm{T}$ is WDI, then the restriction $T \mid X(T,[0,1])$ is also WDI, so we would have the contradiction that $\mathrm{T}$ contains a proper spectral maximal subspace of $X(T,[0,1])$. This shows that the intersection of the classes WDI and $(\delta)$ is a proper subclass of decomposable operators.

We close this section with a more detailed discussion of the relation between WDI and WD operators; we first generalize slightly the notion of WDI.

Definition 3.7. We call $T \in L(X)$ weakly well-decomposable (WWD) if for each finite open cover $\left\{G_{i}: 1 \leq i \leq n\right\}$ of $\mathbf{C}$

(i) Definition 3.1(i) holds and

(ii) there exists a positive integer $\mathrm{m}$ such that for each pair $(j, i)(i=$ $1,2, \ldots, n ; j=1,2, \ldots)$ there is an operator $P_{j i}$ satisfying $C^{m}(T) P_{j i}=0$ and Definition 3.1(ii, a and b).

We claim that Theorem 3.2 and 3.3 remain true for WWD operators. For let $\mathrm{T}$ be a WWD operator on X. In the proof of Theorem 3.2 given above, we need only show that the equality $(\lambda-T) f(\lambda)=0$ implies that $Q_{j} f(\lambda)=0$ for $\lambda \in G_{1} \backslash H_{1}^{-}$. From the equality $(\lambda-T) f(\lambda)=0$ we have

$$
\begin{aligned}
(\lambda-T)^{m} Q_{j} f(\lambda) & =\sum_{k=0}^{m}(-1)^{m-k} T^{m-k} Q_{j} \lambda^{k} f(\lambda) \\
& =\sum_{k=0}^{m}(-1)^{m-k} T^{m-k} Q_{j} T^{k} f(\lambda) \\
& =C^{m}(T) Q_{j} f(\lambda)=0
\end{aligned}
$$


for $\lambda \in G_{1} \backslash H_{1}^{-}$. Then $Q_{j} f(\lambda)=0$ for all such $\lambda$, since $(\lambda-T)^{m}$ is injective. Continuing the argument as in Theorem 3.2, we conclude that T has SVEP. As for Theorem 3.3, we need only verify the inclusion $\sigma_{T}\left(Q_{j} x\right) \subset \sigma_{T}(x)$. Let $\mathrm{x}($.$) be the local resolvent of \mathrm{T}$ at $\mathrm{x}$. Then

$$
(\lambda-T) x(\lambda)=x \quad\left(\lambda \notin \sigma_{T}(x)\right) .
$$

Differentiating (3.1) k times, we obtain

$$
(\lambda-T) x^{(k)}(\lambda)=-k x^{(k-1)}(\lambda) .
$$

Now define the following analytic function on $C \backslash \sigma_{T}(x)$ :

$$
y(\lambda)=\sum_{k=0}^{m-1}(-1)^{k} C^{k}(T) Q_{j} \frac{x^{(k)}(\lambda)}{k !} .
$$

Then

$$
\begin{aligned}
T y(\lambda) & =\sum_{k=0}^{m-1}(-1)^{k} T C^{k}(T) Q_{j} \frac{x^{(k)}(\lambda)}{k !} \\
& =\sum_{k=0}^{m-1}(-1)^{k} C^{k+1}(T) Q_{j} \frac{x^{(k)}(\lambda)}{k !}+\sum_{k=0}^{m-1}(-1)^{k} C^{k}(T) Q_{\jmath} T \frac{x^{(k)}(\lambda)}{k !}
\end{aligned}
$$

hence for $\lambda \notin \sigma_{T}(x)$, from (3.2) and $C^{m}(T) Q_{j}=0$,

$$
\begin{aligned}
(\lambda-T) y(\lambda) & =\sum_{k=0}^{m-1}(-1)^{k} C^{k}(T) Q_{j}(\lambda-T) \frac{x^{(k)}(\lambda)}{k !} \\
& -\sum_{k=0}^{m-1}(-1)^{k} C^{k+1}(T) Q_{j} \frac{x^{(k)}(\lambda)}{k !} \\
& =Q_{j}(\lambda-T) x(\lambda)+\sum_{k=1}^{m-1}(-1)^{k+1} C^{k}(T) Q_{j} \frac{x^{(k-1)}(\lambda)}{(k-1) !} \\
& +\sum_{k=0}^{m-1}(-1)^{k+1} C^{k+1}(T) Q_{j} \frac{x^{(k)}(\lambda)}{k !}=Q_{j} x
\end{aligned}
$$

which proves the result. Thus $\mathrm{X}(\mathrm{T}, \mathrm{F})$ is closed for all closed $\mathrm{F}$.

The following example shows that there are WWD operators which are not WDI.

Example 3.8. Let $T_{1}$ be the operator defined in $[4$, p. 26(2)] and denote the domain of $T_{1}$ by $Y$. Then it was proved in [4, pp. 26-27] that $T_{1}$ is WD 
but not decomposable relative to the identity. Now let $T_{2}$ be the operator in Ex. 3.5 acting on Z. Then $T=T_{1} \oplus T_{2}$ on $X=Y \oplus Z$ is WWD, but we now show that T is not WDI. By [4, p. 27] $Y=Y_{0} \oplus Y_{1}$ (in [4, p. 27], $Y, Y_{0}, Y_{1}$ are denoted $X, X_{0}, X_{1}$ resp.). Suppose that $\mathrm{T}$ is WDI. By Definition 3.1, there exists $\left\{P_{j}\right\} \subset\{T\}^{\prime}$ such that each $x \in X$ with $\sigma_{T}(x) \subset\{\lambda:|\lambda| \leq 1 / 3\}$ can be realized as

$$
\text { WOT }-\lim _{j \rightarrow \infty} P_{j} x=x,
$$

and all $x \in X$ with $\sigma_{T}(x) \subset\{\lambda:|\lambda| \geq 2 / 3\}$ satisfies

$$
P_{j} x=0 \text {. }
$$

Let $Q \in L(X)$ be the projection of X onto Y along Z, and put $R_{j}=Q P_{j} Q \mid Y$. Then $R_{j}$ commutes with $T_{1}$, and hence by [1, Prop. 2.6]

$$
R_{j}(f, g)=\left(a_{j} f+b_{j} \partial g+c_{j} g, a_{j} g\right) \text { for all }(f, g) \in Y,
$$

where $a_{j}, b_{j}, c_{j} \in Y_{0}$ and $a_{j}$ is analytic on the interior of the unit disc D. If $(0, g) \in Y$ with $\sigma_{T_{1}}((0, g)) \subset\{\lambda:|\lambda| \geq 2 / 3\}$, then $R_{j}(0, g)=0$ by $(3.4)$; hence $a_{j}=0$ by analyticity. It follows from (3.5) that

$$
R_{j}(f, g)=\left(b_{j} \partial g+c_{j} g, 0\right) \text { for all }(f, g) \in Y .
$$

Choose now $(f, 0) \in Y$ with $\sigma_{T_{1}}((f, 0)) \subset\{\lambda:|\lambda| \leq 1 / 3\}$ and $f \neq 0$. Then (3.3) and (3.6) imply that

$$
(f, 0)=\text { WOT }-\lim _{j \rightarrow \infty} R_{j}(f, 0)=0 .
$$

This contratiction proves that $\mathrm{T}$ is not WDI. But $\mathrm{T}$ cannot be WD either because $T_{2}$ is not decomposable by Ex. 3.5.

The previous example shows that the class of WWD operators properly contains the class of WDI operators as well as the WD operators.

\section{Automatic continuity.}

Theorem 4.1. Let $T \in L(X)$ have property $(\delta)$ and let $S \in L(Y)$ be WDI. Then (i) and (ii) are equivalent.

(i) Every linear $\theta: X \rightarrow Y$ that is a generalized intertwining of $T$ with $S$ is bounded.

(ii) $(T, S)$ has no critical eigenvalue and either $T$ is algebraic or $E_{S}(\varnothing)=$ $\{0\}$.

The implication (i) $\Rightarrow$ (ii) always holds by $[17$, Lemma 3.2 and Theorem 3.6]. The proof of the converse relies on the following lemmas. 
Lemma 4.2. If $T$ is algebraic, then $\sigma(T)$ is finite, hence $T$ is decomposable.

Proof. Let $p(\lambda)$ be a nonzero polynomial such that $p(T)=0$. The spectral mapping theorem implies that $p(\sigma(T))=\{p(\lambda): \lambda \in \sigma(T)\}=\sigma(p(T))=$ $\{0\}$, hence $\sigma(T)$ is contained in the finite set of zeros of $p(\lambda)$.

The next lemma is an analog of [14, Proposition 4].

Lemma 4.3. Suppose $S$ is WDI on $X$ and $F$ is closed in $\mathbf{C}$. Then

(4.1) $\cap_{V \supset F}\left[X\left(S, V^{-}\right)+E_{S}(\varnothing)\right] \subset E_{S}(F) \subset \cap_{V \supset F}\left[X\left(S, V^{-}\right)+E_{S}(\varnothing)\right]$.

Proof. Since $X\left(S, V^{-}\right)+E_{S}(\varnothing) \subset E_{S}\left(V^{-}\right)$and since $\cap_{V \supset F} E_{S}\left(V^{-}\right)=E_{S}(F)$ by [11, Theorem 2.6], the first inclusion in (4.1) is immediate. To prove the second inclusion, let $\mathrm{V}$ be open with $F \subset V$ and let $\mathrm{U}$ be open such that $F \subset U \subset U^{-} \subset V$. Let $P_{j}, Q_{j} \in\{S\}^{\prime}$ be operators satisfying Definition 3.1(ii) for the cover $\left\{V, \mathbf{C} \backslash U^{-}\right\}$of $\mathbf{C}$, i.e. $P_{j}+Q_{j} \rightarrow I($ WOT $)$ and $P_{j} X \subset X\left(S, V^{-}\right)$ and $Q_{j} X \subset X(S, \mathbf{C} \backslash U)$ for all $j$. For $\lambda \notin F$,

$$
(\lambda-S) Q_{j} E_{S}(F)=Q_{j} E_{S}(F),
$$

so we obtain $Q_{j} E_{S}(F) \subset E_{S}(F)$ by the maximality of $E_{S}(F)$. On the other hand,

$$
Q_{j} E_{S}(F) \subset Q_{j} X \subset X(S, \mathbf{C} \backslash U) \subset E_{S}(\mathbf{C} \backslash U) .
$$

From [11, Theorem 2.6] again,

$$
Q_{j} E_{S}(F) \subset E_{S}(F) \cap E_{S}(\mathbf{C} \backslash U)=E_{S}(\varnothing) .
$$

Thus for each $x \in E_{S}(F)$ and $u \in X^{*}$, the equality

$$
\langle x, u\rangle=\lim \left\langle\left(P_{j}+Q_{j}\right) x, u\right\rangle
$$

implies that $x \in\left[X\left(S, V^{-}\right)+E_{S}(\varnothing)\right]^{-}$. This proves the second inclusion.

Corollary 4.4. Let $S$ be WDI. If $E_{S}(\varnothing)=\{0\}$, then $E_{S}(F)=X(S, F)$ for each closed $F$, and thus $S$ is admissible.

Proof. The condition $E_{S}(\varnothing)=\{0\}$ together with (4.1) shows $E_{S}(F)=$ $X(S, F)$. So Theorem 3.3 shows that $\mathrm{S}$ is admissible.

Proof of (ii) $\Rightarrow$ (i) of Theorem 4.1. If (T, S) has no critical eigenvalue and $E_{S}(\varnothing)=\{0\}$, we use Corollary 4.4 and [13, Corollary 9] to reach (i). If $(T, S)$ has no critical eigenvalue and $T$ is algebraic, then we may assume 
that $\mathrm{S}$ has a nontrivial divisible subspace (otherwise we are reduced to the case $E_{S}(\varnothing)=\{0\}$ ), hence we may use Lemma 4.2 and [15, Corollary 3.3] to reach (i).

Corollary 4.5. Assume $T \in L(X)$ has property $(\delta)$ and $S$ is the WDI operator in Example 3.5. Then any linear generalized intertwining of $T$ with $S$ is continuous.

Proof. By Theorem 4.1 it suffices to prove that (T, S) has no critical eigenvalue and $E_{S}(\varnothing)=\{0\}$. The former is evident since $S$ has no eigenvalues. To prove the latter, let $\mathrm{L}$ be any linear manifold such that $(\mu-S) L=L$ for all $\mu$. We prove $L=\{0\}$. Let $|\mu|<1$. If $y \in L, y \neq 0$, then $y=\left(f_{j}\right)$ with $f_{j} \in B^{j}(D)$, and there is $u \in L$ with $u=\left(g_{j}\right)$ and $y=(\mu-S) u$. Hence $(\mu-S) g_{j}(z)=f_{j}(z)$ for all $j$ and $|z| \leq 1$. We may suppose that $f_{k}(\lambda)=1$ for some $k$ and $|\lambda| \leq 1$. But then $g_{k}$ is discontinuous at $z=\lambda$, contradicting the construction of S.

Remark 1. Lemma 4.3 remains valid for WWD operators. Let $\mathrm{S}$ be such an operator, and let $\mathrm{F}, \mathrm{U}, \mathrm{V}$ be the sets in Lemma 4.3. Assume $P_{j}, Q_{j}$ are the operators satisfying Definition 3.7(ii). Then $P_{j}+Q_{j} \rightarrow I$ (WOT), $P_{j} X \subset$ $X\left(S, V^{-}\right)$and $Q_{j} X \subset X(S, \mathbf{C} \backslash U)$ for all $j$. By [15, Cor. 1.2] $Q_{j} E_{S}(F) \subset$ $E_{S}(F)$, hence $Q_{j} E_{S}(F) \subset X(S, \mathbf{C} \backslash U) \cap E_{S}(F) \subset E_{S}(\mathbf{C} \backslash U) \cap E_{S}(F)=$ $E_{S}(\varnothing)$. Hence the second inclusion in (4.1) follows, and since the first inclusion is evident, we conclude that Lemma 4.3, Theorem 4.1 and Cor. 4.4 all hold for WWD operators.

Remark 2. In [9, Theorem 1] and [10, Theorem 1.2.1] the authors proved the following theorem.

Theorem 4.6. Let $T \in L(X)$. Then $T$ is decomposable if and only if for every open cover $\{G, H\}$ of $\mathbf{C}$ there is a linear map $P: X \rightarrow X$ such that

(i) if $x \in X$, then $\sigma_{T}(P x) \subset G^{-}$and $\sigma_{T}(x-P x) \subset H^{-}$;

(ii) if $F$ is closed in $G \backslash H^{-}$and $x \in X(T, F)^{-}$, then $P x=x$;

(iii) if $K$ is closed in $H \backslash G^{-}$and $x \in X(T, K)^{-}$, then $P x=0$.

According to $[13$, p. 329$]$ it is still an open question whether a decomposable operator with no nontrivial divisible subspaces is admissible. But if the linear map $\mathrm{P}$ in Theorem 4.6 commutes with $\mathrm{T}$, then Lemma 4.3 and hence Corollary 4.4 remain valid: $\mathrm{T}$ is admissible. Here the problem reduces to that of constructing a $\mathrm{P}$ satisfying (i)-(iii) of Theorem 4.6 and commuting with $\mathrm{T}$. 


\section{Further considerations.}

In this section we give several applications of the foregoing results. We say that an operator is "weakly subdecomposable relative to the identity" if it is the restriction of a WDI operator to an invariant subspace; "subscalar" operator is a similar restriction of a generalized scalar operator.

Theorem 5.1. Assume $T \in L(X)$ has property $(\delta)$ and $S \in L(Y)$ is weakly subdecomposable relative to the identity. If $(T, S)$ has no critical eigenvalue, $E_{V}(\varnothing)=\{0\}$ and $S=V \mid Y$ for some WDI operator $V \in L(W)$, then every generalized intertwining of $T$ with $S$ is continuous.

Proof. Corollary 4.4 and Proposition 2.7 imply that $\mathrm{S}$ is admissible. The theorem now follows from [13, Corollary 9].

Corollary 5.2. Assume $T$ has property $(\delta)$ and $S$ is subscalar. If $S$ has no eigenvalue, then every generalized intertwining of $T$ with $S$ is continuous. In particular, if $S$ is hyponormal with no eigenvalue, then every intertwining of $T$ with $S$ is continuous.

Proof. Let $V \in L(W)$ be a generalized scalar extension of $\mathrm{S}$. Then $\mathrm{V}$ is WDI [5, p. 94] and $E_{V}(\varnothing)=\{0\}$. The last equality follows from a result of Vrbova [18] that

$$
W(V, F)=\cap\left\{(\lambda-V)^{p} W: \lambda \notin F\right\}
$$

for some fixed $p>0$. Let $\mathrm{Z}$ be a linear manifold with $(\lambda-V) Z=Z$ for all $\lambda$. Then

$$
Z=(\lambda-V)^{p} Z \subset(\lambda-V)^{p} W \quad(\lambda \in \mathbf{C}) .
$$

Hence $Z \subset W(V, \varnothing)$ by (5.1). This shows $E_{S}(\varnothing)=\{0\}$, so the conclusion follows from Theorem 5.1. The last assertion follows from Putinar's theorem [16] that hyponormal operators are subscalar.

Our final result requires knowing that the generalized scalar operator $\mathrm{T}$ is "completely regular" [5, p. 110] if for every $A \in L(X)$ the condition $A X(T, F) \subset X(T, F)$ for each closed $\mathrm{F}$ implies that A commutes with one of the spectral distributions of $\mathrm{T}$ (hence $\mathrm{A}$ and $\mathrm{T}$ commute).

Proposition 5.3. Let $T \in L(X)$ be a completely regular generalized scalar operator, and suppose that $T$ has no eigenvalues. If $\theta$ is a generalized intertwining of $T$, then $\theta$ commutes with $T$.

Proof. By Corollary $5.2 \theta \in L(X)$, hence by [5, Theorem 4.4.5] $\theta X(T, F) \subset$ $X(T, F)$ for every closed $\mathrm{F}$ in $\mathrm{C}$. Thus $\theta E_{T}(F) \subset E_{T}(F)$ for every such 
F, because by Corollary 4.4 and previous proof the algebraic and analytic spectral manifolds of a generalized scalar operator agree on the closed sets in $\mathbf{C}$ (hence $E_{T}(\varnothing)=\{0\}$ ). The complete regularity. of $\mathrm{T}$ yields the desired conclusion.

\section{References}

[1] E. Albrecht, An example of a $C^{\infty}(\mathbf{C})$-decomposable operator which is not $C^{\infty}(\mathbf{C})$ spectral, Rev. Roumaine Math. Pures Appl., 19 (1974), 131-139.

[2] _ An example of a weakly decomposable operator which is not decomposable, Rev. Roumaine Math. Pures Appl., 20 (1975), 855-861.

[3] - On two questions of I, Colojoara and C. Foias, Manuscripta Math., 25 (1978), 1-15.

[4] E. Albrecht, J. Eschmeier and M.M. Neumann, Some topics in the theory of decomposable operators, Operator Theory, Advances and applications , Birkhauser, Basel, 17 (1986), 15-34.

[5] I. Colojoara and C. Foias, Theory of generalized spectral operators, Gordon and Breach, New York, 1968.

[6] I. Erdelyi and S. Wang, A local spectral theory for closed operators, LMS Lecture Note Series 105, Cambridge, 1985.

[7] A. Jafarian, Weakly and quasidecomposable operators, Rev. Roumaine Math. Pures Appl., 22 (1977), 195-212.

[8] R. Lange, On generalization of decomposability, Glasgow Math. J., 22 (1981), 7781.

[9] R. Lange and S. Wang, Universal notions characterizing spectral decompositions, Glasgow Math. J., 34 (1992), 109-116.

[10] - New approaches in spectral decomposition, Contemporary Math., 128, Providence, 1992.

[11] K.B. Laursen, Algebraic spectral subspaces and automatic continuity, Czech. Math. J., 38(113) (1988), 157-172.

[12] K.B. Laursen and M.M. Neumann, Decomposable operators and automatic continuity, J. Operator Theory, 15 (1986), 33-51.

[13] - Automatic continuity of intertwining linear operators on Banach spaces, Rend. del Circolo Mat. di Palermo, serie II, XL (1991), 325-341.

[14] K.B. Laursen and P. Vrbova, Some remarks on the surjectivity spectrum of linear operators, Czech Math. J., 39(114) (1989), 730-839.

[15] M.M. Neumann, Decomposable operators and generalized intertwining linear transformations, Operator Theory, Advances and applications, Birkhauser, Basel, 28(c) (1988), 209-222.

[16] M. Putinar, Hyponormal operators are subscalar, J. Operator Theory, 12 (1984), 385-395.

[17] A.M. Sinclair, Automatic continuity of linear operators, LMS Lecture Note Series 21, Camridge, 1971.

[18] P. Vrbova, The structure of the maximal spectral spaces for generalized scalar operators, Czech. Math. J., 23(98) (1973), 493-496. 
[19] S. Wang and I. Erdelyi, The spectral decomposition property of the sum and product of two commuting operators, Tohoku Math. J., 41 (1989), 657-672.

Received June 16, 1990 and revised November 16, 1994.

HAMPTON UNIVERSITY

HAMPTON, VA 23668-0001

AND

NANJING UNIVERSITY

NANJING, 210008

P.R. ChINA

AND

DALHOUSIE UNIVERSITY

Halifax, Nova Scotia

CANADA, B3H 3J5

E-MAIL ADDRESS: ZLONG@CS. DAL.CA 



\title{
PACIFIC JOURNAL OF MATHEMATICS
}

Founded by E. F. Beckenbach (1906-1982) and F. Wolf (1904-1989)

\section{EDITORS}

\author{
Sun-Yung Alice Chang (Managing Editor) \\ University of California. \\ Los Angeles, CA 90095-1555 \\ pacific@math.ucla.edu
}

\section{F. Michael Christ}

University of California

Los Angeles, CA 90095-1555

christ@math.ucla.edu

Thomas Enright

University of California

San Diego, La Jolla, CA 92093

tenright@ucsd.edu

Nicholas Ercolani

University of Arizona

Tucson, AZ 85721

ercolani@math.arizona.edu
Robert Finn

Stanford University

Stanford, CA 94305

finn@gauss.stanford.edu

Vaughan F. R. Jones

University of California

Berkeley, CA 94720

vfr@math.berkeley.edu

Steven Kerckhoff

Stanford University

Stanford, CA 94305

spk@gauss.stanford.edu
Martin Scharlemann

University of California

Santa Barbara, CA 93106

mgscharl@math.ucsb.edu

Gang Tian

Courant Institute

New York University

New York, NY 10012-1100

tiang@taotao.cims.nyu.edu

\section{S. Varadarajan}

University of California

Los Angeles, CA 90095-1555

vsv@math.ucla.edu

\section{SUPPORTING INSTITUTIONS}

\section{CALIFORNIA INSTITUTE OF TECHNOLOGY NEW MEXICO STATE UNIVERSITY \\ OREGON STATE UNIVERSITY \\ STANFORD UNIVERSITY \\ UNIVERSITY OF ARIZONA \\ UNIVERSITY OF BRITISH COLUMBIA \\ UNIVERSITY OF CALIFORNIA UNIVERSITY OF HAWAII}

\author{
UNIVERSITY OF MONTANA \\ UNIVERSITY OF NEVADA, RENO \\ UNIVERSITY OF OREGON \\ UNIVERSITY OF SOUTHERN CALIFORNIA \\ UNIVERSITY OF UTAH \\ UNIVERSITY OF WASHINGTON \\ WASHINGTON STATE UNIVERSITY
}

The supporting Institutions listed above contribute to the cost of publication of this Journal, but they are not owners or publishers and have no responsibility for its contents or policies.

Manuscripts must be prepared in accordance with the instructions provided on the inside back cover.

The Pacific Journal of Mathematics (ISSN 0030-8730) is published monthly except for July and August. Regular subscription rate: $\$ 215.00$ a year (10 issues). Special rate: $\$ 108.00$ a year to individual members of supporting institutions.

Subscriptions, orders for back issues published within the last three years, and changes of subscribers address should be sent to Pacific Journal of Mathematics, P.O. Box 4163, Berkeley, CA 94704-0163, U.S.A. Prior back issues are obtainable from Kraus Periodicals Co., Route 100, Millwood, NY 10546.

The Pacific Journal of Mathematics at the University of California, c/o Department of Mathematics, 981 Evans Hall, Berkeley, CA 94720 (ISSN 0030-8730) is published monthly except for July and August. Second-class postage paid at Berkeley, CA 94704, and additional mailing offices. POSTMASTER: send address changes to Pacific Journal of Mathematics, P.O. Box 6143, Berkeley, CA 94704-0163.

\author{
PUBLISHED BY PACIFIC JOURNAL OF MATHEMATICS at University of California, \\ Berkeley, CA 94720, A NON-PROFIT CORPORATION \\ This publication was typeset using AMS-LATEX, \\ the American Mathematical Society's TEX macro system. \\ Copyright (C) 1995 by Pacific Journal of Mathematics
}




\section{PACIFIC JOURNAL OF MATHEMATICS}

Volume $171 \quad$ No. $1 \quad$ November 1995

Generalized fixed-point algebras of certain actions on crossed products

BEATRIZ ABADIE

Partitions, vertex operator constructions and multi-component KP

equations

MAarten Bergvelt and A. P. E. Ten Kroode

Holomorphy tests based on Cauchy's integral formula

CARMEN CASCANTE and DANIEL PASCUAS

The Euler characteristic of a nonpositively curved, piecewise Euclidean manifold

RUTH CHARNEY and MiChaEl WALTER DAVIS

The invariant connection of a $\frac{1}{2}$-pinched Anosov diffeomorphism and rigidity

RENATO FERES

The inverse Riemann mapping theorem for relative circle domains

ZHENG-XU HE and OdED SCHRAMM

Multipliers and Bourgain algebras of $H^{\infty}+C$ on the polydisk

KeI Ji IZUCHI and YASOU MATSUGU

Irreducible bimodules associated with crossed product algebras. II

TSUYOSHI KAJIWARA and SHIGERU YAMAGAMI

Elliptic fibrations on quartic $K 3$ surfaces with large Picard numbers

MASATO KUWATA

Automatic continuity for weakly decomposable operators

RIDGLEY LANGE, SHENG-WANG WANG and YONG ZHONG

Crosscap number of a knot

Hitoshi MURAKAMI and AKIRA YASUHARA

Symmetric minimal surfaces in $\mathbb{R}^{3}$ 